\title{
Modified Billroth-I reconstruction after distal gastrectomy
}

\author{
Yoshiyuki Hoya ${ }^{1}$, Tetsuya Taki ${ }^{1}$, Masato Hoshino ${ }^{1}$, Atsuo Shida ${ }^{1}$, Shuzou Kohno ${ }^{1}$, Tomoyoshi Okamoto ${ }^{1}$, \\ and KATSUHIKO YANAGA ${ }^{2}$ \\ ${ }^{1}$ Department of Surgery, Daisan Hospital, The Jikei University School of Medicine, 4-11-1 Izumihon-cho, Komae, Tokyo 201-8601, Japan \\ ${ }^{2}$ Department of Surgery, The Jikei University School of Medicine, Tokyo, Japan
}

To the Editor:

Although Billroth I (B-I) or Billroth II (B-II) has traditionally been the method for reconstruction after distal gastrectomy, Roux-en-Y (R-Y) is now being increasingly employed at many institutions, mainly to prevent duodenogastric reflux and to ensure safe anastomosis. We do perform R-Y in our department, but only in limited cases in patients with special conditions, i.e., locally invasive tumors in the pylorus or the antrum in which the tumor may recur locally, or in patients having a subtotal distal gastrectomy for which a gastroduodenal anastomosis would result in excessive tension. The advantages of R-Y after distal gastrectomy include the absence of or a low incidence of reflux gastritis and esophagitis [1] and a possible reduction in the future development of stump carcinoma [2]. On the other hand, with R-Y, stomal ulcer may develop $[3,4]$ and the incidence of cholelithiasis, for which endoscopic sphincterotomy is almost impossible, may increase $[5,6]$. The notorious Roux stasis syndrome [7] may also develop in some cases.

The advantages of B-I over R-Y include food passage through the physiological route, single anastomosis, low risk of stomal ulcer or cholelithiasis, and easy access to the duodenal papilla in case there is cholelithiasis or pancreatic disease. Thus, we select B-I reconstruction whenever possible.

Because the main problem with B-I is duodenogastric reflux, we recently developed a modified B-I technique, in which the gastric mucosa is inverted like a checkvalve in the duodenum to prevent reflux (Fig. 1). Since we first reported the technique in 2007 [8], ten such patients have been followed up for a mean of 17.7

Offprint requests to: Y. Hoya

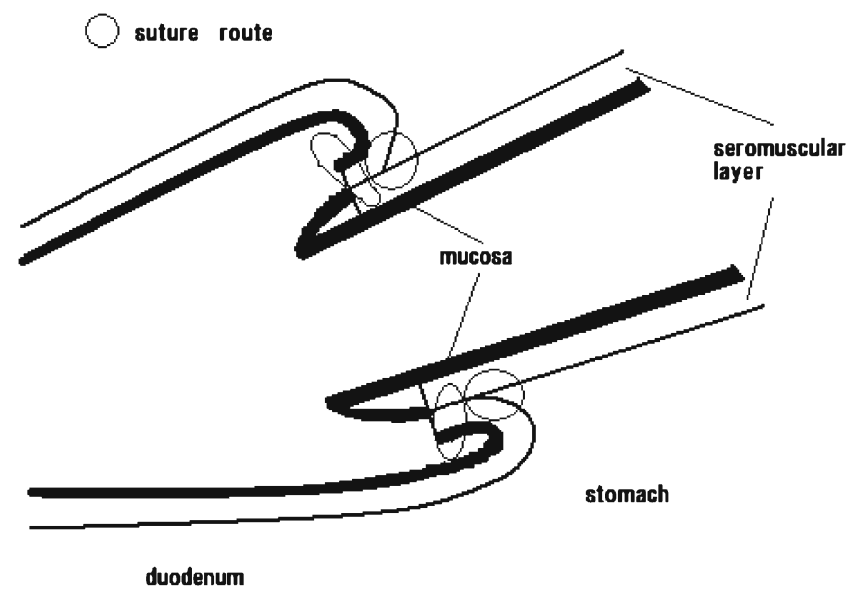

Fig. 1. Schematic view of the newly developed modified Billroth I technique. With permission from the publishers of Surgery [8]

months (range, 8-30 months). Follow-up endoscopy always showed a clear gastric stump with no or little bile (Fig. 2). We expect this technique to be tested by many gastric surgeons.

\section{References}

1. Collard JM, Romagnoli R: Roux-en-Y jejunal loop and bile reflux. Am J Surg 2000;179:298-303.

2. Ma Z, Wang Z, Zhang J. Carcinogenicity of duodenogastric reflux juice in patients undergoing gastrectomy. Zhonghua Wai Ke Za Zhi (Chinese Journal of Surgery) 2001;39:764-6.

3. Oliver JV. Effect of vagus section and isolation of the pyloric antrum on the development of the Mann-Williamson ulcer in the dog. Surg Forum 1953;4:336-8.

4. Turnage RH, Sarosi G, Cryer B, Spechler S, Peterson W, Feldman $M$. Evaluation and management of patients with recurrent peptic ulcer disease after acid-reducing operations: a systematic review. J Gastrointest Surg 2003;7:606-26. 


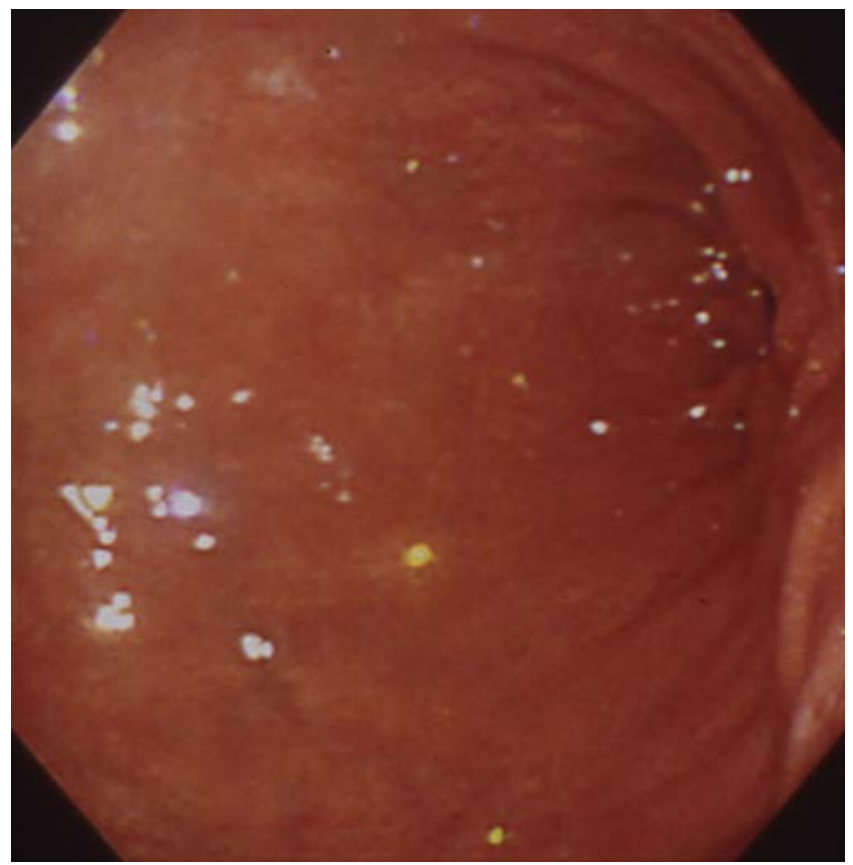

5. Akatsu T, Yoshida M, Kubota T, Shimazu M, Ueda M, Otani Y, et al. Gallstone disease after extended (D2) lymph node dissection for gastric cancer. World J Surg 2005;29:182-6.

6. Kobayashi T, Hisanaga M, Kanehiro H, Yamada Y, Ko S, Nakajima Y. Analysis of risk factors for the development of gallstones after gastrectomy. Br J Surg 2005;92:1399-403.

7. Le Blanc-Louvry I, Ducrotte P, Lemeland JF, Metayer J, Denis P, Teniere P. Motility in the Roux-Y limb after distal gastrectomy: relation to the length of the limb and the afferent duodenojejunal segment: an experimental study. Neurogastroenterol Motil 1999; 11:365-74.

8. Hoya Y. Modified Billroth I after distal gastrectomy for prevention of remnant gastritis? The feasibility report (Billroth I evolved in year 120$)$. Surgery $2007 ; 142: 427-8$.

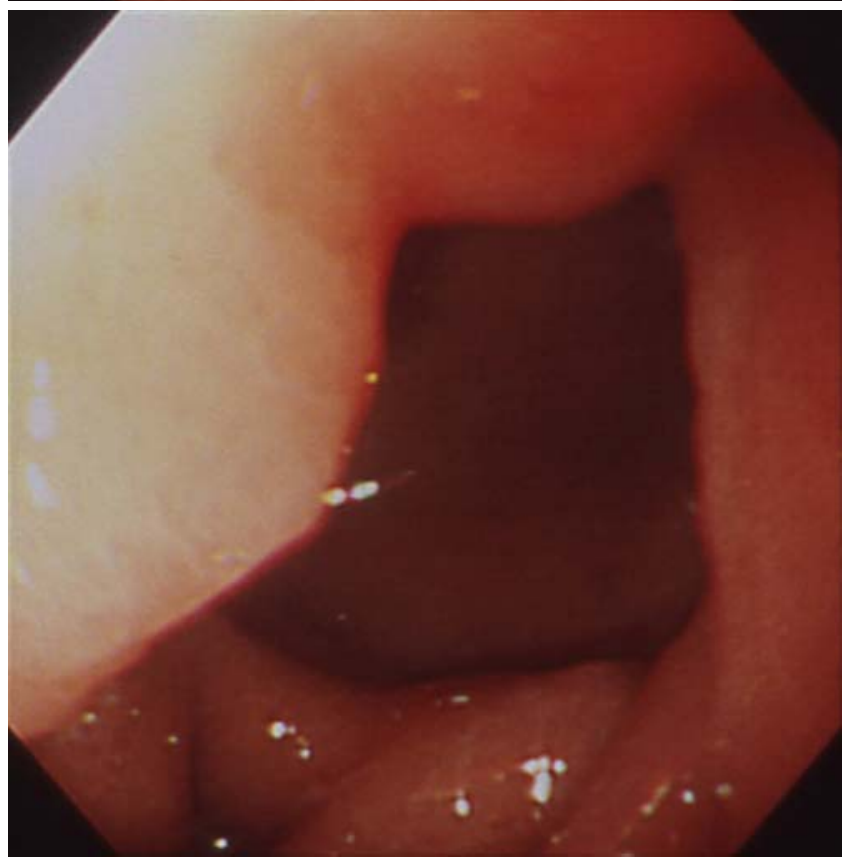

Fig. 2. Endoscopic pictures of the remnant stomach and anastomosis after modified Billroth-I reconstruction 